\title{
Current Application of Functional/Targeted Echocardiogram in NICU: An Update
}

\author{
Rezoana Rima1, Mohammad Abdullah Al Mamun ${ }^{2}$ \\ Department of Paediatric Cardiology, Bangladesh Institute of Child Health and Dhaka Shishu \\ (Children) Hospital, Dhaka
}

\begin{abstract}
:
Keywords: $\quad$ Functional echocardiograph is the bedside use of cardiac ultrasound to monitor functional and Echocardiography, hemodynamic changes longitudinally. It can assist in an understanding of pathophysiology of the Haemodynamic, $N I C U$ rapidly changing neonatal hemodynamic status including cardiac function and systemic and pulmonary blood flow in critically ill preterm and term neonates. It also facilitates the evaluation of response to therapeutic intervention. This review examines the potential applications of functional echocardiography in different disease states, and how the technology may be introduced safely in the NICU.
\end{abstract}

(Cardiovasc. j. 2017; 10(1): 74-83)

\section{Introduction:}

Newborn may have a variety of haemodynamic problems with a variable and complex pathophysiology that at times have limited clinical manifestations. This cardiovascular vulnerability stems from particular features of the newborn, such as incomplete myocardial development, presence of foetal shunts, changes in systemic and pulmonary vascular resistance and complex hemodynamic changes that take place during transition to extra uterine life. Despite progressive technological advances in neonatal intensive care, haemodynamic monitoring in newborns is still based on assessment of continuous heart rate, blood pressure (BP), acid base status, urine output and capillary refill time. ${ }^{1}$ These measures provide important and useful information to the clinician. Clinical assessment and the classic parameters mentioned before to elucidate the underlying pathophysiology in haemodynamic disturbances can sometime lead to incorrect assumptions and therapeutic decisions, which may even cause harm. ${ }^{2}$ Situations in which serial assessment is needed to evaluate response to treatment or the haemodynamic changes that take place during transition from foetal to postnatal circulation, it is important to consider that these changes can be very different from what might be assumed clinically based on conventional assessment. ${ }^{1}$ These variables associated in varying degrees to tissue perfusion, which is the key haemodynamic parameter, and for which an adequate monitoring method has yet to be developed. ${ }^{3}$

Standard methods of cardiovascular monitoring in the neonate have limitations. Sometimes the clinical signs provide limited insight into the adequacy of systemic blood flow and organ perfusion. Enhanced cardiovascular imaging and hemodynamic evaluation offers novel insights regarding the contribution of the ductus arteriosus, myocardial performance and pulmonary hemodynamics to ongoing clinical instability. In addition, it allows more accurate delineation of the nature of the underlying disease process and facilitates the evaluation of response to therapeutic intervention. ${ }^{4}$ The term functional echocardiography have been introduced to describe the echocardiography as an adjunct in clinical assessment of the hemodynamic status in neonate. It can provide non invasive information on the underlying CVS pathology causing hemodynamic instability and response to treatment. It is not intended as a substitute for the evaluation of a neonate with suspected CHD by qualified paediatric cardiologist.

Address of Correspondence: Dr. Rezoana Rima, Department of Paediatric Cardiology, Bangladesh Institute of Child Health and Dhaka Shishu (Children) Hospital, Dhaka, Bangladesh. E-mail: drrezoana@gmail.com 


\section{Application in NICU}

Assessment of myocardial function, hypovolemia and organ perfusion

End-organ perfusion is dependent on systemic blood flow and vascular resistance; therefore, reliance on blood pressure measurements alone provides limited information regarding the adequacy of organ blood flow. ${ }^{5}$ The poor correlation between blood pressure and left ventricular output in the first day of life emphasizes this point. ${ }^{6}$ In addition there is a weak relationship between blood pressure and superior vena cava (SVC) flow which represents upper body circulation including the brain. ${ }^{7}$ Both blood pressure and systemic flow are important determinants of the likelihood of altered perfusion, and neither should be monitored nor treated in isolation, without consideration of the influence of the other. Serial echocardiography offers the potential of novel insights as to the physiologic nature of the cardiovascular impairment: specifically, whether the concern relates to preload, afterload or myocardial contractility.

\section{Evaluation of cardiac output}

Doppler assessment of left (LVO) and right (RVO) ventricular outputs provides additional hemodynamic information regarding the adequacy of blood flow. Assessment of LVO involves measuring the mean velocity of blood flow across the ascending aorta from an apical five-chamber view using PW
Doppler (Fig.1) and determining the diameter of the aortic root from a parasternal long-axis view using $\mathrm{m}$-mode methods. The area under the wave form of the aortic systolic beat can be used to calculate the velocity time integral (VTI), which is a measure of the distance traveled by blood during a given beat. Multiplying that by the aortic cross-sectional area (AoCSA) derived from Ao CSA $=\mathrm{pr}^{2} / 4$ ( $\mathrm{r}=$ aortic diameter) gives the stroke volume. LVO can be derived from multiplying stroke volume by heart rate: $\mathrm{LVO}=($ AoCSA X VTI X heart rate $) /$ weight and is expressed in $\mathrm{ml} / \mathrm{kg} / \mathrm{min}$. Normal values range from 170 to $320 \mathrm{ml} / \mathrm{kg} / \mathrm{min} .{ }^{8}$ Walther et al. ${ }^{9}$ evaluated cardiac output in normal healthy term \& preterm neonates during $1^{\text {st }}$ week of life by pulse Doppler echocardiography. They have showed mean cardiac output values $( \pm \mathrm{SD})$ per kilogram of body weight were $249 \pm 34 \mathrm{~mL} / \mathrm{mm} / \mathrm{kg}$ and decreased with advancing birth weight (Table I). LVO must be used with caution as it may give a falsely reassuring picture of acceptable systemic blood flow in the presence of hemodynamically significant ductus arteriosus (HSDA) specifically, as a high volume left-to-right shunt will lead to increased pulmonary venous return, left heart preload and stroke volume, but at the expense of systemic blood flow. Early measurement of LVO following PDA ligation has been shown to predict late-onset impairment in LV contractility, low systolic arterial pressure and need for cardiotropes. ${ }^{10}$
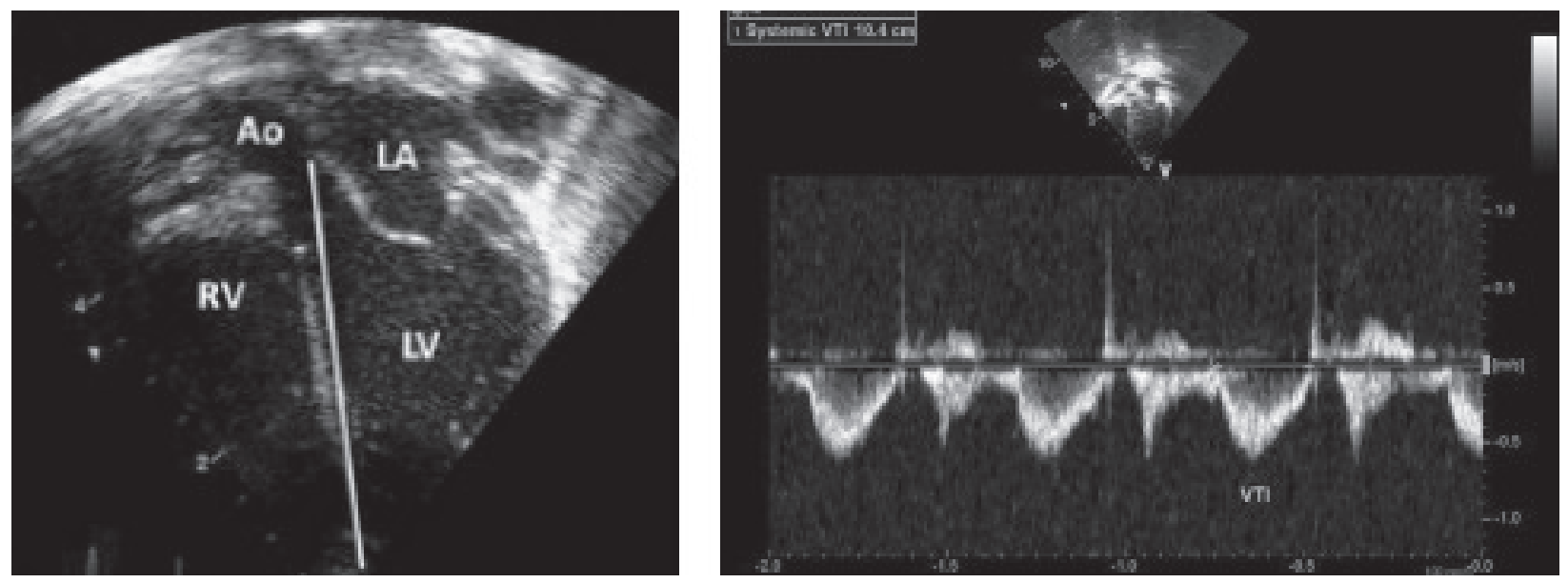

Fig.-1: Showing Pulsed-wave Doppler measures blood flow across the aortic valve (white lines). The area under the curve is then traced to obtain the velocity time integral (dotted line). $R V$, right ventricle; $L V$, left ventricle; Ao, aorta; LA, left atrium. 
Table-I

Cardiac output of neonates at different birth weight ${ }^{9}$

\begin{tabular}{lc}
\hline Birth weight & Cardiac output \\
\hline$<1,500 \mathrm{~g}$ & $265 \pm 32 \mathrm{~mL} / \mathrm{min} / \mathrm{kg}$ \\
1,500 to $2,500 \mathrm{~g}$ & $253 \pm 34 \mathrm{~mL} / \mathrm{min} / \mathrm{kg}$ \\
$>2,500 \mathrm{~g}$ & $241 \pm 33 \mathrm{~mL} / \mathrm{min} / \mathrm{kg}$ \\
\hline
\end{tabular}

Measurement of RVO is done using a similar approach described above. The pulmonary artery diameter is best assessed from an oblique long axis parasternal view. The RV VTI is obtained from Doppler interrogation of the same view. RVO reflects blood return from the systemic circulation and, in the absence of a large trans-atrial shunt, is reflects systemic blood flow in infants. However, RVO measurements are confounded by the presence of atrial shunt. Typically the atrial shunts are much smaller than ductal shunts, therefore RVO may be used as an estimation of systemic blood flow. 6,8

SVC flow has been proposed to be a better echocardiographic measure of systemic blood flow as it reflects exclusive venous return from the brain and upper body and is untainted by shunts. SVC flow can be assessed using echocardiography. ${ }^{11} \mathrm{~A}$ subcostal approach is used to assess SVC Doppler signals and a high supra- sternal view is used to measure SVC diameter.

The SVC flow was calculated using the following formula: $\mathrm{SVC}$ flow $=$ (velocity time integral X $(\mathrm{p} \mathrm{X}$ (mean SVC diameter2/4) X heart rate)/body weight. The resulting figure is expressed as $\mathrm{ml} / \mathrm{kg} / \mathrm{min}$. Normal values $40-120 \mathrm{ml} / \mathrm{kg} / \mathrm{min}$ in VLBW.As fourfifths of flow in the SVC represents venous return from the head and neck, such measurements may provide novel insights into any association between regional cerebral blood flow and cerebral injury. Reduced flow in the SVC is common in premature infants in the first $24 \mathrm{~h}$, reaching a nadir between 8 and $12 \mathrm{~h}$ which coincides with increased systemic vascular resistance. Low SVC return is associated with an increased incidence of late intraventricular hemorrhage and may be one factor in the causal pathway of impaired preterm neurodevelopmental outcome. ${ }^{12,13}$ However SVC return reflects blood supply to the brain and upper body and therefore no information regarding blood supply to the liver, kidney and gut can be derived. Second, cerebral blood flow is subjected to intrinsic autoregulatory mechanisms and may not truly reflect cardiac performance. ${ }^{14}$ Measurement of SVC diameter beyond $48 \mathrm{~h}$ of life is difficult. In addition, the diameter of the vessel varies widely within the cardiac cycle. Therefore a wide margin of error would have resulted from volumetric calculations. Groves et al. ${ }^{15}$ have also questioned the validity of this measurement due to high interobserver variability.

Inferior Vena Caval (IVC) diameter \& variation in IVC provides indirect evidence of intravascular volume which is important for diagnosis of neonatal hypotension \& shock. An average size of IVC $<8 \mathrm{~mm}$ in term $\&<6 \mathrm{~mm}$ in preterm is considered as rough estimate of hypovolemia. Also more than 50\% collapsibility of IVC is suggestive of hypovolemia.

\section{Evaluation of myocardial performance}

\section{Systolic performance}

Maturational differences make the immature myocardium susceptible to impaired performance. These developmental disadvantages make the neonatal myocardium vulnerable during a hypoxic ischemic insult or when subjected to preload or afterload compromise. The evaluation of myocardial contractility is challenging as current methods are load dependent, although serial evaluation may provide valuable insights. The immature neonate is particularly sensitive to sudden changes in afterload. ${ }^{16}$ The immediate transitional period after elimination of the placenta from the systemic circulation and following PDA ligation represent clinical situations in which afterload may be playing an important role.

Left ventricular (LV) systolic performance can be assessed by shortening fraction (SF), ejection fraction or the rate-corrected mean velocity of circumferential fibre shortening $(\mathrm{mVCF})$. SF characterizes LV contractility using m-mode methods from either a long parasternal axis or short axis view of the left ventricle (Fig.-2). It is calculated by measuring left ventricular end-diastolic diameter (LVEDD) and left ventricular end-systolic diameter (LVESD) using m-mode echo and applying the following formula:

$$
\mathrm{SF} \%=(\text { LVEDD }- \text { LVESD) X100/ (LVEDD) }
$$

Normal neonatal values for SF\% are $28-40 \%$. Shortening fraction is an unreliable measure of systolic LV function in the first few days of life as high right ventricular pressures impair ventricular septal wall movement. ${ }^{17}$ 


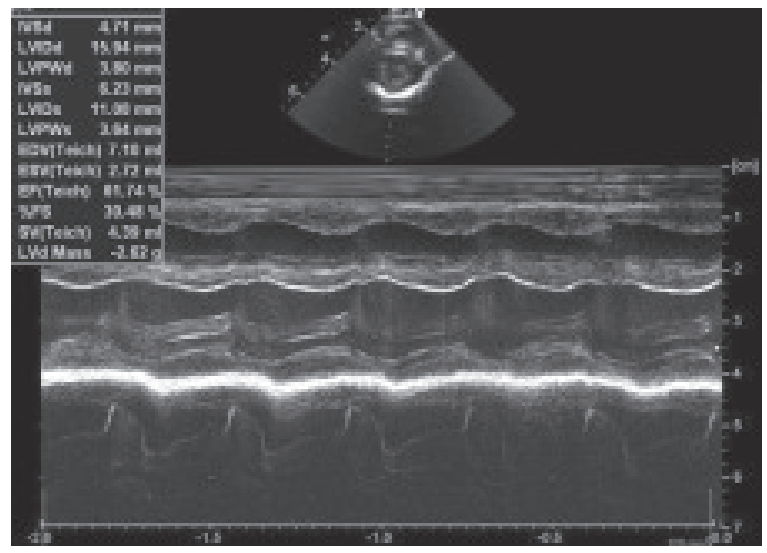

Fig.-2: M-Mode measure of shortening fraction

LV mean velocity of circumferential fractional shortening $(\mathrm{mVCFc})$ is a preload-independent afterload adjusted measure of LV function. It is determined by the following method ${ }^{18}$ :

$$
\mathrm{mVCFc}=(\text { LVEDC }- \text { LVESC }) /(\text { LVEDC X ETc })
$$

Where LVEDC is left ventricular end diastolic circumference, LVESC is left ventricular end-systolic circumference, and ETc is left ventricular ejection time corrected for heart rate (ET/ÖRR interval, where $R R$ is the time between consecutive heart beats).

The stress velocity relationship is a load-independent measure of myocardial contractility ${ }^{19}$ where the relationship between $\mathrm{mVCFc}$ and end-systolic wall stress analysis is examined by linear regression methods. The clinical applicability of these markers is not well studied in neonates. However, there is some evidence that $\mathrm{mVCFc}$ correlates with cardiac troponin levels in the early neonatal period. ${ }^{20}$ Tissue Doppler methods, speckled tracking and other novel methods of analysis have been studied in adults but their application in neonate's remains poorly understood.

\section{Diastolic performance}

Diastolic performance is altered in the newborn but its relevance to clinical decision-making is not wellappreciated. Diastolic filling is influenced by the compliance of the ventricular wall. In adults and older children, transmitral flow occurs in two phases: an early phase of passive flow during which the majority of the filling occurs and a late atrial contraction phase during which the remaining third of the venous return to the ventricle is delivered. In patients with diastolic dysfunction, the majority of filling occurs during the atrial phase as the stiff ventricular wall prevents passive early flow across the mitral valve. The immature fetal and preterm myocardium is characterized by impaired diastolic function. PW Doppler can be used to examine this biphasic pattern of transmitral flow. First phase is the early wave ( $\mathrm{E}$ wave) and is a result of passive blood flow across the atrioventricular valves in early diastole. The second phase is the atrial contraction wave (A wave) and is a result of atrial contraction at the end of diastole. Maximum $\mathrm{E}$ and $\mathrm{A}$ wave velocities are compared as ratios (Fig.-3). An E:A ratio $<1$ indicates diastolic dysfunction (indicating a non-compliant ventricle). Transmitral VTI can be measured to give an estimate of flow across the atrioventricular valves. ${ }^{21}$

The Tei index (or myocardial performance index) has been found to be a valuable quantitative echocardiography index of ventricular function by incorporating both systolic and diastolic performance of the right and left ventricles (Fig.-4). It is defined as the sum of isovolumetric contraction and relaxation times divided by the ejection time, which requires measurement of the time interval between
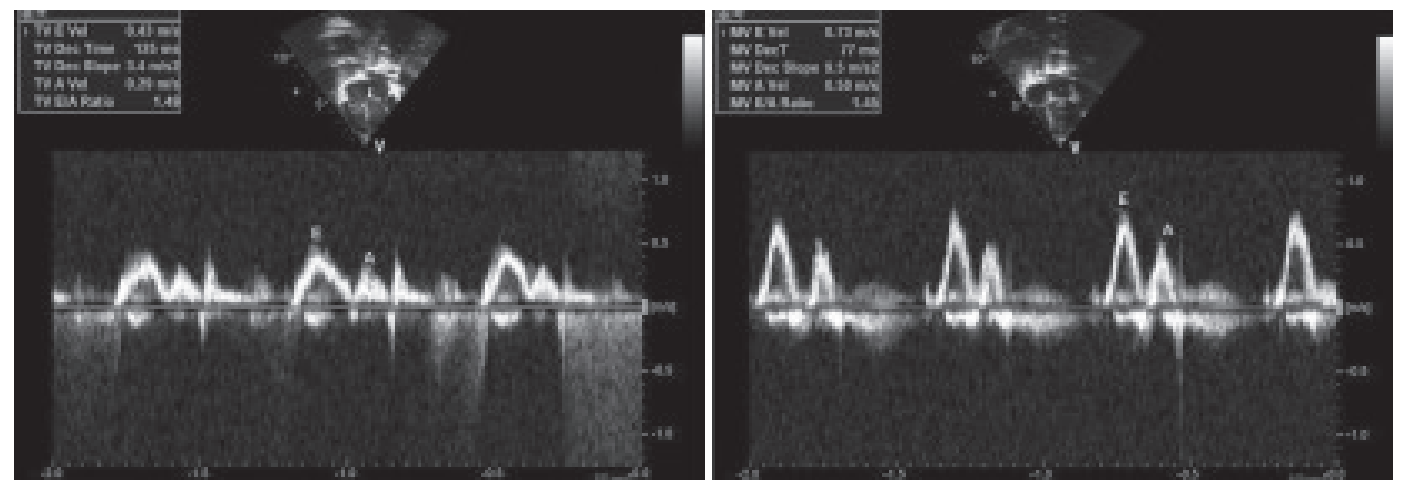

Fig.-3: Assessment of diastolic function. Doppler traces of transmitral (right) and tricuspid valves (left) can be obtained. The early wave (E wave, passive diastolic blood flow) and atrial wave (A wave, flow due to atrial contraction) are measured. 
the end and onset of mitral or tricuspid inflow (the ' $a$ ' interval) and the ejection time of the LV or RV outflow (the 'b' interval). ${ }^{22}$ The Tei index is then calculated by the formula $(\mathrm{a}-\mathrm{b}) / \mathrm{b}$. The Tei index is easy to calculate, reproducible, and independent of heart rate and blood pressure. Normal values in healthy neonates range from 0.25 to $0.38 .{ }^{23} \mathrm{It}$ is relatively independent of age and also has a low degree of inter and intra observer variability. ${ }^{24}$

\section{Hemodynamic assessment of a PDA and clinical relevance}

Prior to echocardiography, the diagnosis of PDA was reliant on the evolution of clinical signs including:

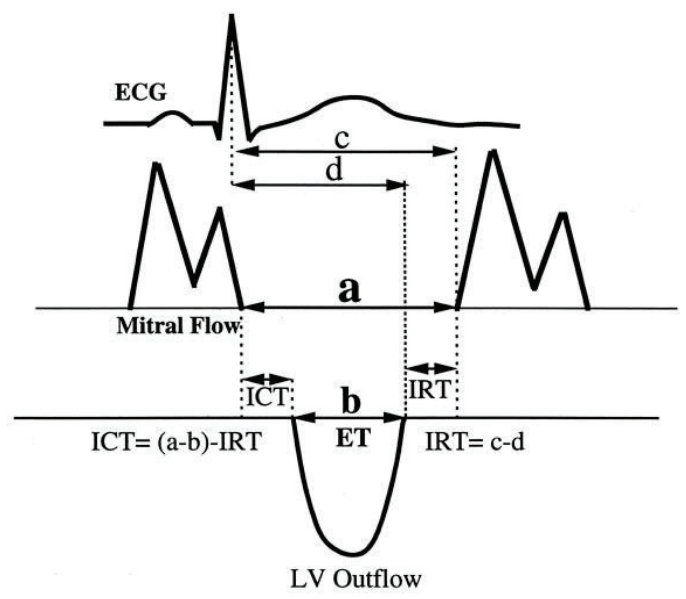

Index $=\frac{\mathbf{a}-\mathbf{b}}{\mathbf{b}}=\frac{(\mathrm{ICT}+\mathrm{IRT})}{\mathbf{E T}}$

Fig.-4: TEI measurement of ventricle. a murmur, increased precordial activity, widened pulse pressure and bounding peripheral pulses. However, the diagnosis of a significant PDA by echocardiography precedes the development of clinical signs by a mean of 2 days. ${ }^{25}$ There is poor correlation between physical signs and the presence of PDA by echo in the first week of life. ${ }^{26,27}$ Bounding pulses and a murmur may be absent in up to $20 \%$ of infants with a PDA. ${ }^{28}$ Therefore echocardiography remains the gold standard for PDA diagnosis. ${ }^{26}$ Measurement of the internal ductal diameter by two-dimensional and colour Doppler imaging allows early prediction of significant PDA in preterm infants. Although a transductal diameter $>1.5 \mathrm{~mm}$ has a good predictive value of ductal persistence, the measurement is not without limitation. ${ }^{29}$ The ductus is a dynamic vessel of variable architecture, with an unpredictable response to treatment. It is not possible to directly quantify the magnitude of transductal flow, but the impact on the pulmonary and systemic circulations is measurable.

Pulmonary over circulation attributable to excessive transductal flow and the effect of ductal steal on systemic perfusion may also be quantified using echocardiography for a comprehensive evaluation of the significance of the ductal shunt shown in Table-II. ${ }^{4}$

\section{Evaluation of severity of pulmonary hypertension}

Assessment of right ventricular systolic pressure (RVSP) is possible in the presence of tricuspid

Table-II

Surrogate markers of significant PDA. ${ }^{4}$

\begin{tabular}{llcc}
\hline Measurement & Modality \& sample gate & Moderate PDA & Large PDA \\
\hline Ductus arteriosus & & & \\
Diameter (mm) & High parasternal ductal view & $1.5-3.0$ & $>3.0$ \\
Ductal velocity (m/s) & PWD at ductal view(PA) & $1.5-2.0$ & $>1.5$ \\
PA diastolic flow (m/s) & PWD at left PA & $0.3-0.5$ & $>0.5$ \\
Pulmonary overcirculation & m-Mode: long axix view & $1.5-1.7$ & $>1.7$ \\
LA: Ao ratio & Doppler:transmitral view & $1.0-1.5$ & $>1.5$ \\
E wave: A wave ratio & PWD between MV \& AV & $35-45$ & $<35$ \\
IVRT $(\mathrm{ms})$ & & & \\
Systemic hypoperfusion & PWD at LV outflow tract & $200-300$ & $>300$ \\
Left ventricular output (m//Kg/min) & PWD at beyond PDA & $30-50$ & $>50$ \\
Diastolic descending Ao flow (\%) & PWD at flow at SVC & $<2.4$ & $>2.4$ \\
LVO/SVC ratio & PWD at celiac artery & $0.10-0.15$ & $<0.10$ \\
Celiac artery flow: LVO ratio &
\end{tabular}

PDA, patent ductus arteriosus; PWD, pulsed-wave Doppler; LA, left atrium; Ao, aorta; IVRT, isovolumic relaxation time; MV, mitral valve; AV, aortic valve; LV, left ventricle; LVO, left ventricular output; SVC, superior vena cava. 
regurgitation and calculated using the Bernoulli equation (Fig.-5 \& 6):

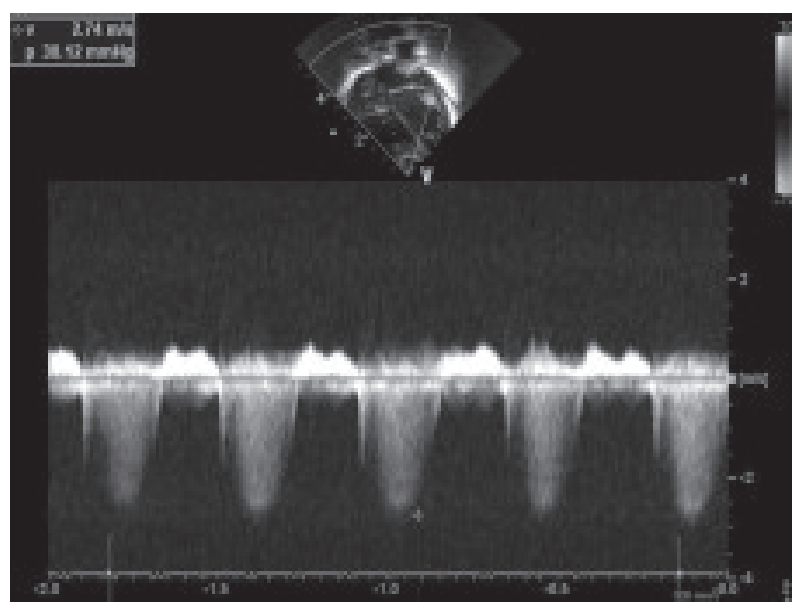

Fig.-5: Showing Continuous wave Doppler of tricuspid regurgitation. The regurgitant jet is characterized by turbulent flow depicted by a full Doppler envelope (upper panel). The peak velocity of the regurgitant jet was $2.74 \mathrm{~m} / \mathrm{s}$, resulting in a pressure gradient of $30 \mathrm{mmHg}$.

RVSP $=$ right atrial pressure $+(4 \times$ Tricuspid Regurgitation JET Vmax; Where Vmax = peak velocity

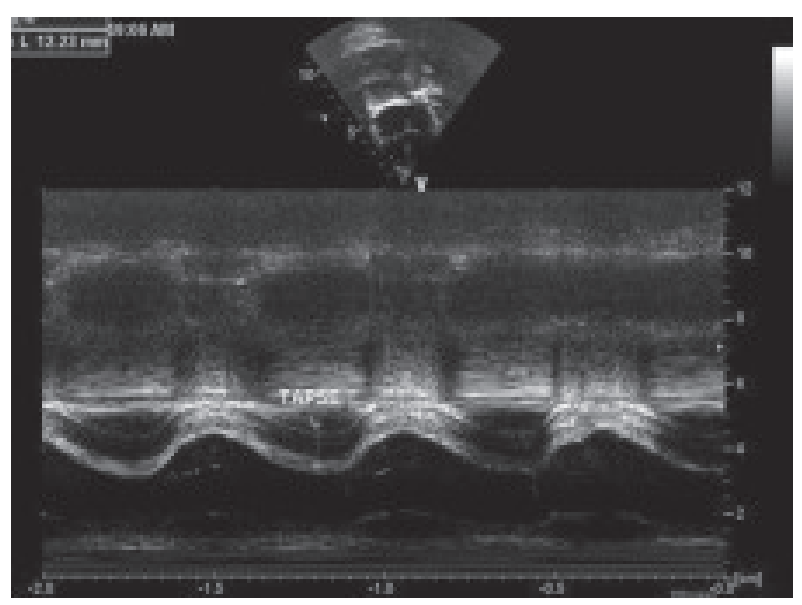

Fig.-6: Tricuspid annular plane systolic excursion (TAPSE).In 4 chamber view a straight line (M mode) is drawn through the lateral tricuspid valve annulus (arrow 1). The level of excursion of the tricuspid valvar plane during systole (TAPSE in $\mathrm{mm}$ ) corresponds with $R V$ ejection fraction (arrow 2). $5 \mathrm{~mm}=20 \% R V E F, 10 \mathrm{~mm}=30 \% R V E F, 15 \mathrm{~mm}$ $=40 \% R V E F, 20 \mathrm{~mm}=50 \% R V E F$.
Table-III

Grading of pulmonary hypertension

\begin{tabular}{lccc}
\hline Grade 1(Mild) & $\begin{array}{c}\text { Systolic } \\
(\mathrm{mm} \mathrm{Hg}) \\
30-50\end{array}$ & $\begin{array}{c}\text { Diastolic } \\
(\mathrm{mm} \mathrm{Hg}) \\
20-25\end{array}$ & $\begin{array}{c}\text { Mean } \\
(\mathrm{mm} \mathrm{Hg}) \\
>20\end{array}$ \\
\hline Grade 2(moderate) & $50-70$ & $26-35$ & $>40$ \\
Grade 3(severe) & $70-110$ & $36-45$ & $>50$ \\
$\begin{array}{l}\text { Grade 4(systemic or } \\
\text { suprasystemic) }\end{array}$ & $>110$ & $46-55$ & $>60$ \\
\hline
\end{tabular}

This measurement will underestimate the severity of the pulmonary hypertension if there is impaired RV contractility. The presence of PDA can also be used to estimate pulmonary pressures if systemic systolic pressures are known. Pulmonary arterial pressure can be estimated in the presence of a PDA with unrestrictive flow. The peak velocity of transductal flow can be applied to the Bernoulli equation to calculate the pressure difference between the systemic and pulmonary circulations. The direction of transductal flow may also be used to evaluate the severity of the pulmonary hypertension. A pure unrestrictive right-to-left shunt is seen with suprasystemic pulmonary hypertension; whereas a bidirectional shunt implies that pulmonary artery pressures approximate systemic pressures. The percentage of right-to-left flow of total transductal flow may also be calculated to further document the severity of the pulmonary hypertension.

In the absence of these shunts, direct assessment of pulmonary artery pressures is difficult in premature infants, particularly in those patients with chronic lung disease. Assessment of pulmonary artery using the ratio of pulmonary artery ejection time to right ventricular ejection time (AT/RVET(C)), which can be estimated noninvasively from Doppler pulmonary artery wave forms, has been shown to correlate negatively with pulmonary artery pressures in premature infants. ${ }^{29-31}$ Acceleration time is measured as the time interval between the Doppler signal baseline to peak velocity. RVET is the time interval of the right ventricular ejection phase. The AT/RVET(c) is calculated by dividing AT/RVET by the square root of the $R-R$ interval from a simultaneous electrocardiogram tracing. Several studies have shown that early pulmonary hypertension in premature infants, as assessed by AT/RVET, is a good predictor of late-onset CLD. ${ }^{29}$ 


\section{Evaluation of right ventricular performance and pulmonary hemodynamics}

In some patients the impact of pulmonary hypertension and increased RV afterload may include impaired RV contractility. Although moderate to severe RV systolic dysfunction is obvious by direct visual inspection there is no reliable or early marker of $\mathrm{RV}$ failure. Low $\mathrm{RV}$ output $(<170 \mathrm{ml} / \mathrm{min} / \mathrm{kg})$ may also support a diagnosis of impaired RV performance. The complexity of RV anatomy and the fact that it is 'wrapped' around the LV makes quantitative evaluation by $\mathrm{m}$-mode unfeasible. The RV myofiber architecture is composed of superficial circumferential and dominant deep longitudinal layers aligned base to apex that allow for greater longitudinal than radial shortening and minimum twisting and rotational movements. The longitudinal shortening is the dominant deformation of the RV that provides the major contribution to stroke volume during systole. Tricuspid annular plane systolic excursion (TAPSE) can be measured by "2-dimensional echocardiography-guided M-mode recordings from the apical 4-chamber view, with the cursor placed at the free wall of the tricuspid annulus estimates RV function (Fig.- $7 \& 8$ ).

\section{Utility of Functional neonatal echocardiography in the management of neonatal illness}

In many parts of the world, neonatologists have developed the necessary skills to perform limited bedside functional echocardiography assessments as a means to enhance the care of critically ill babies. ${ }^{30}$ Fecho may provide hemodynamic information that either complements what is clinically suspected or provides novel physiologic insights. The ability to perform timely functional echocardiography is now considered an integral component of the assessment of the critically ill newborn. ${ }^{32-34}$

In Canada, greater use of targeted neonatal echocardiography has only happened in the last few years, and it is just slowly becoming a part of standard neonatal intensive care unit (NICU) care. Harabor A et al. showed a total of 303 consecutive targeted neonatal echocardiographic studies were performed on 129 neonates. Of the 303 studies, 126 $(41.5 \%)$ resulted in management changes. They have concluded that targeted neonatal echocardiography is a valuable tool in the NICU and can contribute substantially to hemodynamic management in the first week of life, PDA

TAPSE vs. Age

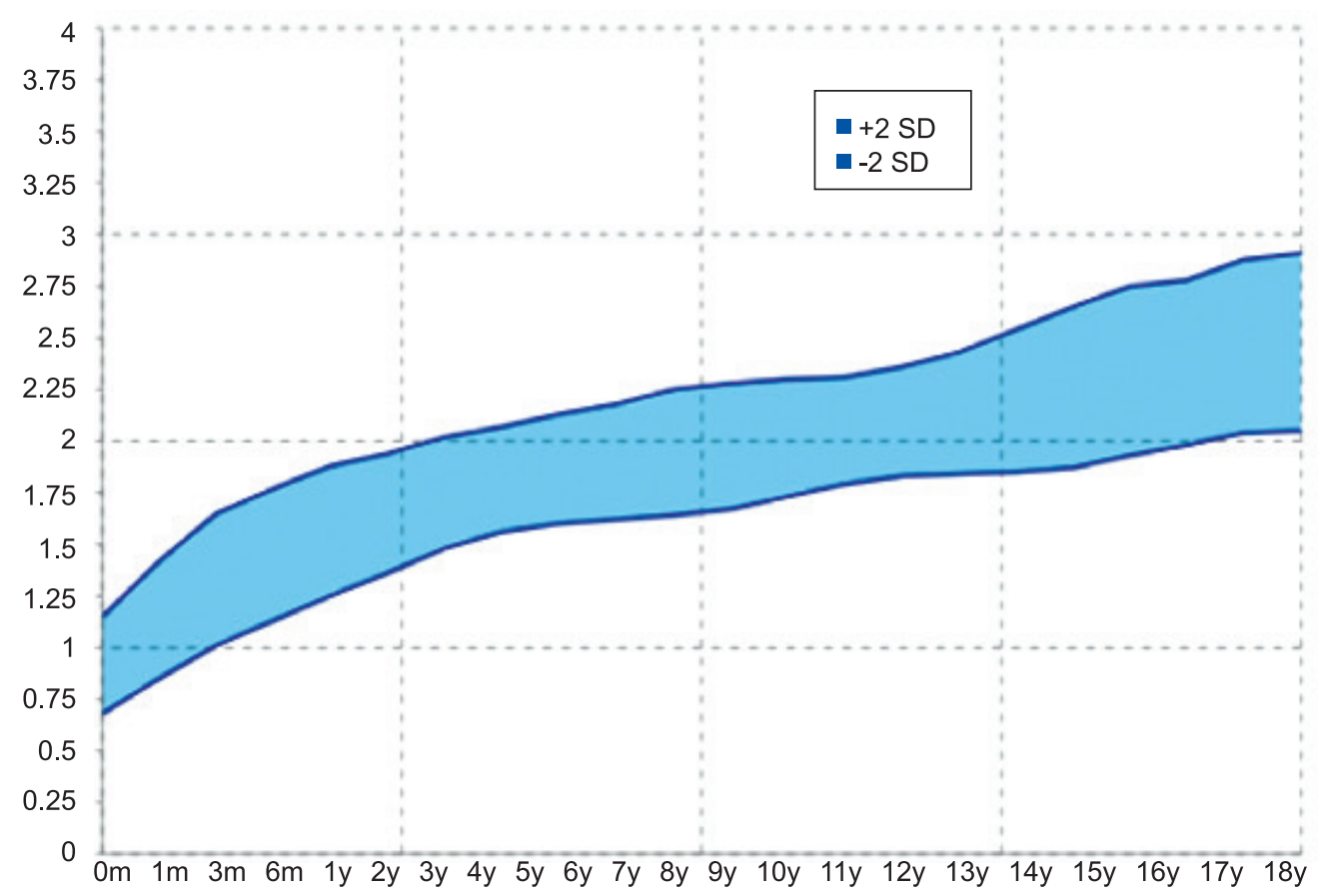

Fig.-7: Showed growth curve TAPSE (mean $\pm 2 S D)$ with age. 
management in the first 2 weeks of life, and cases of hypotension or shock at any time during the hospital stay.

El-Khuffash et al.35reported a retrospective study of targeted neonatal echocardiographic practice at a large referral outborn NICU in Toronto. Despite differences, the impact of targeted neonatal echocardiography was similar in both studies. Khamkar et al. ${ }^{36}$ showed Indian experience of Functional echocardiographic studies of a total of 348 performed in 187 neonates (mean 1.86; SD
2.02). The most frequent indication was Patent Ductus Arteriosus (PDA) assessment ( $\mathrm{n}=174,50 \%)$, followed by haemodynamic instability $(n=43$, 12.36\%). The results of FnECHO modified treatment in 148 cases (42.50\%) in the form of addition and/or change in the treatment or avoidance of unnecessary intervention.

\section{Standards for practice}

There is a growing acceptance that neonatologistperformed functional echocardiography is a useful

\section{Clinical Practice Guidelines: ${ }^{39}$}

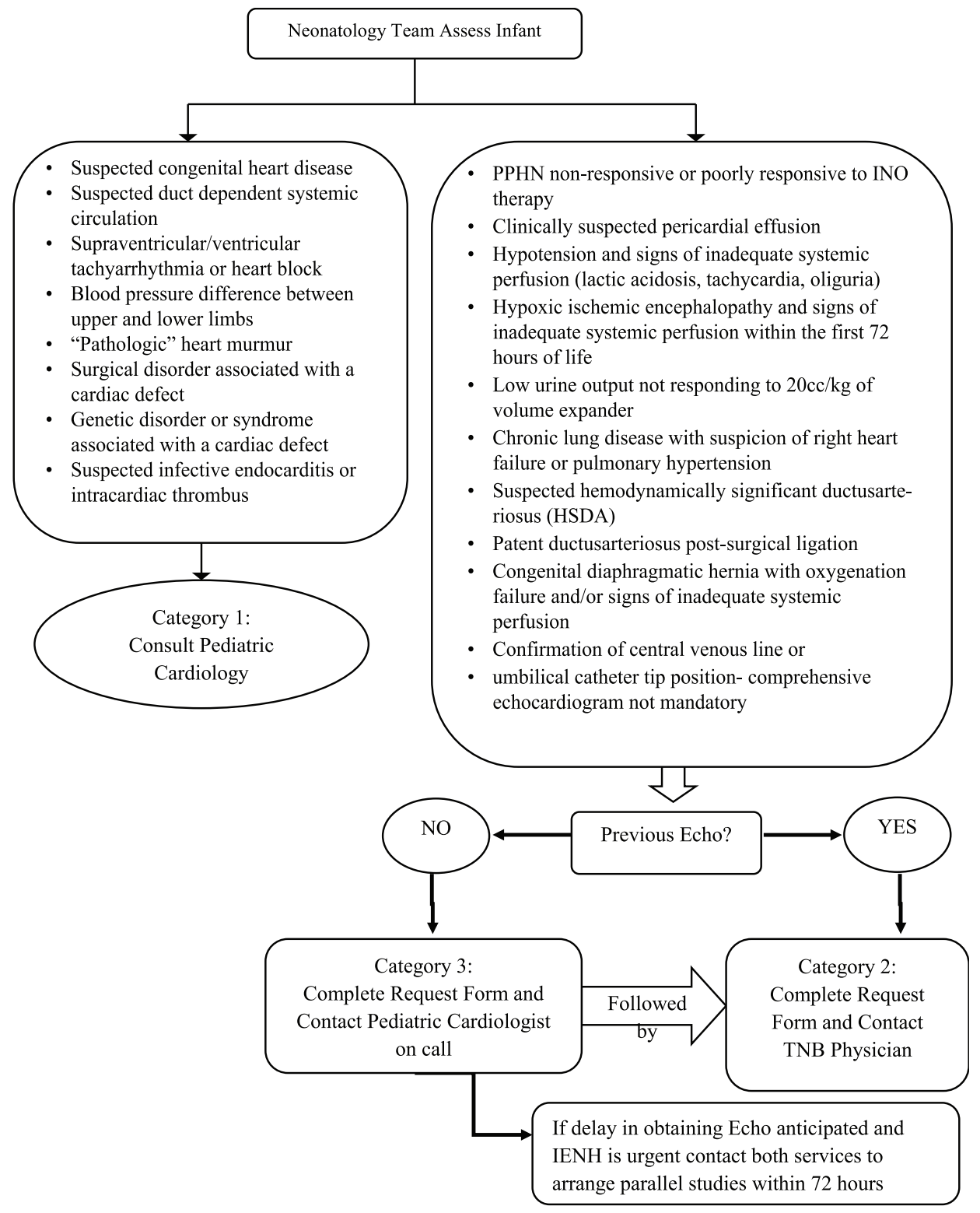

Fig.-8: Echocardiogram Triage Algorithm 
tool in the NICU, with increasing evidence of improved patient outcomes. What is lacking at present is a formalized training and accreditation program necessary for the development of echocardiography skills.37The potential risks of introducing this skill set without formal training include a divergence from clinical assessments and misdiagnosis of congenital heart disease (CHD). This may result in inadvertent withholding of treatments or instituting incorrect therapies. Collaboration with the pediatric cardiology service is essential in developing standards for practice. It is essential that standards are set to ensure that competence is achieved and maintained with ongoing quality assurance. Neonatologists' main concern regarding the use of functional ultrasound by is the potential for misdiagnosis. Although it is important to recognize that CHD can be missed by neonatologists, the presence of a structural cardiac abnormality is almost always detected, even if a full diagnosis is not made. The studies performed by neonatologists serve a different purpose, as alluded to earlier in this review, and should complement those per- formed by cardiologists. Any abnormality detected or suspected should be confirmed by a trained pediatric cardiologist.

The demand for neonatologist-performed functional ultrasound is rising. There are numerous clinicians around the world who use point-of-care functional echocardiography without formal training. There is an urgent need to develop standardized training and accreditation programs to avoid the potential misuse of this skill. Such formal training programs are lacking. This highlights the need for collaboration with pediatric cardiologists in developing these programs as they possess the necessary skills. Formal training programs should include the use of available DVDs, books alongside didactic lectures. Knowledge of practical skills, ultrasound equipment and modalities available also form part of the practical skill set. Emphasis should also be put into recognizing limitations and knowing when to call more experienced staff. These should be conducted by experienced neonatologists and interested pediatric cardiologists. Ongoing education and audit should be an integral part of any training program. ${ }^{38}$ Once the skill set is acquired; logistic challenges need to be addressed. These include access to equipment, study reporting, archiving and storage. Recently Neonatal Patient Care Teams,
HSC \& SBH Child Health Standards Committee developed a neonatal clinical practice guideline for targeted neonatal echocardiography. ${ }^{39}$

\section{Conclusion:}

Functional neonatal echocardiography proved to be a valuable bedside tool for managing neonatal hemodynamics in different NICU around the world. The expanded role of neonatologist-performed functional echocardiography is gaining momentum. There is mounting evidence that it can provide a more comprehensive assessment of the hemodynamic status of infants, and may influence management. There is an urgent need to encourage collaboration with pediatric cardiologists to establish standards for training and maintenance of competency, to develop guidelines for clinical practice and finally to ensure that the necessary clinical governance is in place to clearly identify the complementary roles of the neonatologist and pediatric cardiologist in this process.

\section{Conflict of Interest - None.}

\section{References:}

1. Kluckow M, Seri I, Evans N. Functional Echocardiography: an emerging clinical tool for the neonatologist. J Pediatr 2007; 150:125-130.

2. Sehgal A, McNamara PJ. Does point-of-care functional echocardiography enhance cardiovascular care in the NICU? J Perinatol 2008; 28:729-35.

3. Soleymani S, Borzage M, Seri I. Hemodynamic monitoring in neonates: advances and challenges. $J$ Perinatol 2010;30 Suppl.:S38-45

4. El-Khuffash A, McNamara PJ. Neonatologistperformed functional echocardiography in the neonatal intensive care unit. Seminars in Fetal \& Neonatal Medicine2011; 16: 50-60.

5. Evans JR, Lou SB, Van MK, Cheryl SH. Cardiovascular support in preterm infants. Clin Ther 2006; 28: 13661384.

6. Kluckow M, Evans N. Relationship between blood pressure and cardiac output in preterm infants requiring mechanical ventilation. J Pediatr 1996; 129: 506-12.

7. Kluckow M, Evans N. Low superior vena cava flow and intraventricular haemorrhage in preterm infants. Arch Dis Child Fetal Neonatal Ed 2000; 82: F188-194.

8. Evans N, Kluckow M. Early determinants of right and left ventricular output in ventilated preterm infants. Arch Dis Child Fetal Neonatal Ed 1996; 74: F88e94.

9. Walther FJ, Siassi B, Ramadan NA, Ananda AK, Wu PYK. Pulse Doppler determination of cardiac output in Neonates: Normal standards for clinical use. Peadiatrics 1985; 76: 829-833.

10. Sahni M, Sehgal A, Stewart L, Shivananda S, McNamara PJ. Early functional echocardiography 
(fECHO) predicts postoperative cardiorespiratory instability after patent ductus arteriosus (PDA) ligation. Pediatric Annual Societies Annual Meeting Baltimore E-PAS; 2009:2740.

11. Kluckow M, Evans N. Superior vena cava flow in newborn infants: a novel marker of systemic blood flow. Arch Dis Child Fetal Neonatal Ed 2000; 82: F182e7.

12. Osborn DA, Evans N, Kluckow M. Hemodynamic and antecedent risk factors of early and late periventricular/ intraventricular hemorrhage in premature infants. Pediatrics 2003; 112(1 Pt 1):33e9.

13. Hunt RW, Evans N, Rieger I, Kluckow M. Low superior vena cava flow and neurodevelopment at 3 years in very preterm infants. J Pediatr 2004; 145:588e92.

14. Greisen G. Auto regulation of cerebral blood flow in newborn babies. Early Hum Dev 2005; 81: $423 \mathrm{e} 8$.

15. Groves AM, Kuschel CA, Knight DB, Skinner JR. Echocardiographic assessment of blood flow volume in the superior vena cava and descending aorta in the newborn infant. Arch Dis Child Fetal Neonatal Ed 2008; 93: F24e8.

16. RowlandDG, Gutgesell HP. Noninvasive assessment of myocardial contractility, preload, and afterload in healthy newborn infants. Am J Cardiol 1995; 75: 818e21.

17. Gill AB, Weindling AM. Echocardiographic assessment of cardiac function in shocked very low birth weight infants. Arch Dis Child 1993; 68(1 Special No.): 17e21.

18. Osborn DA, Evans N, Kluckow M. Left ventricular contractility in extremely premature infants in the first day and response to inotropes. Pediatr Res2007; 61:335e40.

19. Colan SD, Borow KM, Neumann A. Left ventricular end-systolic wall stress- velocity of fiber shortening relation: a load-independent index of myocardial contractility. J Am Coll Cardiol 1984; 4: 715e24.

20. El-Khuffash A, Davis PG, Walsh K, Molloy EJ. Cardiac troponin $\mathrm{T}$ and $\mathrm{N}$ - terminal-pro-B type natriuretic peptide reflect myocardial function in preterm infants. J Perinatol 2008; 28: 482e6.

21. Harada K, Shiota T, Takahashi Y, Tamura M, Toyono M, Takada G. Doppler echocardiographic evaluation of left ventricular output and left ventricular diastolic filling changes in the first day of life. Pediatr Res 1994; 35(4 Pt 1):506e9.

22. Tham EB, Silverman NH. Measurement of the Tei index: a comparison of M-mode and pulse Doppler methods. $J$ Am Soc Echocardiogr 2004;17: 1259e65.

23. HaradaK, Tamura M, Toyono M, Yasuoka K. Comparison of the right ventricular Tei index by tissue Doppler imaging to that obtained by pulsed Doppler in children without heart disease. Am J Cardiol 2002; 90: $566 \mathrm{e} 9$.

24. Harjai KJ, Scott L, Vivekananthan K, Nunez E, Edupuganti R. The Tei index: a new prognostic index for patients with symptomatic heart failure. $J \mathrm{Am} S o c$ Echocardiogr2002; 15: 864e8.

25. Skelton R, Evans N, Smythe J. A blinded comparison of clinical and echocar- diographic evaluation of the preterm infant for patent ductus arteriosus. J Paediatr Child Health 1994; 30: 406e11.

26. Alagarsamy S, Chhabra M, Gudavalli M, Nadroo AM, Sutija VG, Yugrakh D. Comparison of clinical criteria with echocardiographic findings in diagnosing PDA in preterm infants. J Perinat Med 2005; 33: 161e4.

27. Evans N. Diagnosis of patent ductus arteriosus in the preterm newborn. Arch Dis Child 1993; 68(1 Special No.): $58 \mathrm{e} 61$.

28. Kupferschmid C, Lang D, Pohlandt F. Sensitivity, specificity and predictive value of clinical findings, $\mathrm{m}$ mode echocardiography and continuous-wave Doppler sonography in the diagnosis of symptomatic patent ductus arteriosus in preterm infants. Eur J Pediatr 1988; $147: 279 \mathrm{e} 82$.

29. Kluckow M, Evans N. Early echocardiographic prediction of symptomatic patent ductusarteriosus in preterm infants undergoing mechanical ventilation. $J$ Pediatr 1995; 127: 774e9.

30. Murase M, Ishida A. Serial pulsed Doppler assessment of pulmonary artery pressure in very low birth-weight infants. Pediatr Cardiol 2000; 21:452e7.

31. Gill AB, Weindling AM. Pulmonary artery pressure changes in the very low birth weight infant developing chronic lung disease. Arch Dis Child 1993; 68(3 Special No.): $303 \mathrm{e} 7$.

32. Subhedar NV, Hamdan AH, Ryan SW, Shaw NJ. Pulmonary artery pressure: early predictor of chronic lung disease in preterm infants. Arch Dis Child Fetal Neonatal Ed 1998; 78: F20e4.

33. Moss S, Kitchener DJ, Yoxall CW, Subhedar NV. Evaluation of echocardiography on the neonatal unit. Arch Dis Child Fetal Neonatal Ed 2003; 88: F287-F291.

34. Skinner JR. Echocardiography on the neonatal unit: a job for the neonatologist or the cardiologist? Arch Dis Child 1998; 78: 401-02.

35. Evans N. Echocardiography on neonatal intensive care units in Australia and New Zealand. J Paediatr Child Health 2000; 36: 169-71.

36. El-Khuffash A, Herbozo C, Jain A, Lapointe A, McNamara PJ. Targeted neonatal echocardiography (TnECHO) service in a Canadian neonatal intensive care unit: a 4-year experience. J Perinatol2013; 33: 687-90.

37. Khamkar AM, Suryawanshi PB, Maheshwari R, Patnaik S, Malshe N, Kalrao V, et al. Functional Neonatal Echocardiography: Indian Experience. Journal of Clinical and Diagnostic Research 2015; 9: SC11-SC14 .

38. Kluckow M, Seri I, Evans N. Functional echocardiography: an emerging clinical tool for the neonatologist. J Pediatr 2007; 150: 125e30.

39. El-Sayed Y, Narvey M, Soni R Baier J, Alvaro R. Integrated Evaluation of Neonatal Hemodynamics (IENH) and Targeted Echocardiogram. Approved by: Neonatal Patient Care Teams, HSC \& SBH Child Health Standards Committee. 